\title{
Circulating Plasma Biomarkers for TSU-68, an Oral Antiangiogenic Agent, in Patients with Metastatic Breast Cancer
}

\author{
Changhoon Yoo, MD 1 \\ Sung-Bae Kim, MD, PhD' \\ Jungsil Ro, MD, $P h D^{2}$ \\ Seock-Ah Im, MD, PhD ${ }^{3}$ \\ Young-Hyuck Im, MD, PhD ${ }^{4}$ \\ Jee Hyun Kim, MD, PhD ${ }^{5}$ \\ Jin-Hee Ahn, MD, PhD' \\ Kyung Hae Jung, $\mathrm{MD}, \mathrm{PhD}^{1}$ \\ Hong Suk Song, MD, $\mathrm{PhD}^{6}$ \\ Seok Yun Kang, MD, $\mathrm{PhD}^{7}$ \\ Hee Sook Park, MD, PhD ${ }^{8}$ \\ Hyun-Cheol Chung, MD, $\mathrm{PhD}^{9}$
}

\footnotetext{
${ }^{1}$ Department of Oncology, Asan Medical Center, University of Ulsan College of Medicine,

Seoul, ${ }^{2}$ Center for Breast Cancer,

National Cancer Center, Goyang,

${ }^{3}$ Department of Internal Medicine,

Seoul National University Hospital, Seoul,

${ }^{4}$ Division of Hematology-Oncology,

Samsung Medical Center,

Sungkyunkwan University School of Medicine,

Seoul, ${ }^{5}$ Department of Internal Medicine,

Seoul National University Bundang Hospital,

Seongnam, ${ }^{6}$ Department of Medicine,

Keimyung University Dongsan Medical Center,

Daegu, ${ }^{7}$ Department of Hematology and

Oncology, Ajou University School of Medicine,

Suwon, ${ }^{8}$ Department of Internal Medicine,

Soonchunhyang University Hospital,

Soonchunhyang University College of Medicine,

Seoul, ${ }^{9}$ Department of Medical Oncology,

Yonsei Cancer Center, Yonsei University

College of Medicine, Seoul, Korea
}

Correspondence: Sung-Bae Kim, MD, PhD Department of Oncology, Asan Medical Center, University of Ulsan College of Medicine, 88 Olympic-ro 43-gil, Songpa-gu, Seoul 05505, Korea

Tel: 82-2-3010-3217

Fax: 82-2-3010-6961

E-mail: sbkim3@amc.seoul.kr

Received March 3, 2015

Accepted May 20, 2015

Published Online July 14, 2015

\section{Purpose}

This study analyzed the role of plasma biomarkers for TSU-68 in a previous phase II trial comparing TSU-68 plus docetaxel and docetaxel alone in patients with metastatic breast cancer.

\section{Materials and Methods}

A total of 77 patients were eligible for this study (38 in the TSU-68 plus docetaxel arm and 39 in the docetaxel alone arm). Blood samples were collected prior to the start of each cycle, and vascular endothelial growth factor (VEGF), platelet-derived growth factor (PDGF)AA, -AB, -BB, fibroblast growth factor, M30, C-reactive protein (CRP), and interleukin 6 (IL-6) levels were measured using enzyme linked immunosorbent assay. The primary endpoint was progression-free survival (PFS).

\section{Results}

In patients with baseline PDGF-AA $\geq$ median, median PFS was significantly worse in the TSU68 plus docetaxel group than in the docetaxel alone group (5.4 months vs. 13.7 months, $p=0.049$ ), while a trend toward a PFS benefit was observed in those with baseline PDGFAA < median (9.7 months vs. 4.0 months, $p=0.18$; $p$ for interaction=0.03). In the TSU-68 plus docetaxel group, PFS showed significant association with fold changes in CRP $(p=0.001)$, IL-6 $(p<.001)$, PDGF-BB $(p=0.02)$, and VEGF $(p=0.047)$ following the first treatment cycle.

\section{Conclusion}

Baseline PDGF-AA levels and dynamics of VEGF, PDGF-BB, CRP, and IL-6 levels were predictive for the efficacy of TSU-68. 


\section{Introduction}

Angiogenesis is a fundamental event in breast cancer growth and metastasis [1], and therefore has been a major target in development of new agents for breast cancer. A number of types of antiangiogenic agents have been investigated in phase III trials in patients with advanced breast cancer [2]. Of these, oral small-molecule multikinase agents, which inhibit diverse angiogenesis targets, including vascular endothelial growth factor receptor (VEGFR) and plateletderived growth factor receptor (PDGFR), have been widely investigated, although their role in management of breast cancer has yet to be established.

TSU-68 (orantinib), a novel oral antiangiogenic multikinase agent, selectively inhibits VEGFR-2, PDGFR, and fibroblast growth factor receptor (FGFR) [3]. Based on promising results from multiple single-arm phase II trials of TSU-68 in patients with breast cancer [4,5], we previously conducted a randomized phase II trial comparing the combination of TSU-68 and docetaxel with docetaxel monotherapy in patients with metastatic breast cancer in whom anthracycline therapy had failed [6].

In the era of targeted therapy, the role of biomarkers has become increasingly important for selection of patients who will benefit most, as the magnitude of benefit from each agent varies among patients. A number of antiangiogenic agents have been approved for management of various types of cancers, although the role of biomarkers remains to be clearly demonstrated for these agents. However, previous studies have suggested the potential value of soluble plasma proteins as pharmacodynamic and predictive biomarkers for antiangiogenic agents [7]. Based on these findings, an exploratory translational study was incorporated into a randomized phase II trial of TSU-68. Candidate markers related to angiogenesis, apoptosis, and inflammation were analyzed for the pharmacodynamics of TSU-68 and prediction of clinical outcomes in breast cancer patients treated with TSU-68containing chemotherapy. Here, we report on the results of this biomarker analysis of TSU-68 in patients with metastatic breast cancer.

\section{Materials and Methods}

\section{Patients, treatment, and outcome}

This study was an exploratory analysis of a multicenter randomized phase II trial comparing TSU-68 plus docetaxel with docetaxel alone in patients with metastatic breast cancer who had previously received therapy with an anthracyclinecontaining regimen. Eligible patients were randomized at a $1: 1$ ratio to receive either docetaxel $60 \mathrm{mg} / \mathrm{m}^{2}$ on day 1 plus TSU-68 $400 \mathrm{mg} / \mathrm{m}^{2}$ on days 1-21 every 3 weeks or docetaxel $60 \mathrm{mg} / \mathrm{m}^{2}$ alone on day 1 every 3 weeks. The primary endpoint was progression-free survival (PFS) by independent review. The details of this study have been published elsewhere [6].

In brief, between November 2006 and December 2007, 77 patients were eligible for analysis (38 for TSU-68 plus docetaxel and 39 for docetaxel alone). The median PFS was 6.8 months (95\% confidence interval [CI], 5.4 to 12.5 months) in the TSU-68 plus docetaxel group and 8.1 months $(95 \% \mathrm{CI}, 4.0$ to 13.7 months) in the docetaxel-alone group (hazard ratio, $1.0 ; 95 \% \mathrm{CI}, 0.6$ to $1.8 ; \mathrm{p}=0.95)$. No significant differences in the overall response rates and overall survival were observed between groups ( $p=0.29$ and $p=0.42$, respectively). In subgroup analyses for prespecified patient groups (anthracycline sensitive/resistant, prior taxanes/non-prior taxanes, estrogen receptor positive/negative, progesterone receptor positive/ negative, HER2 positive/ negative, triple negative), no differences in PFS was observed across all subgroups between arms. This study was approved by the institutional review boards of each participating institution and conducted in accordance with the Declaration of Helsinki and Good Clinical Practice guidelines. All participants provided written informed consent before enrollment.

\section{Plasma collection and enzyme-linked immunosorbent assay assays}

Blood samples were collected at baseline and prior to the start of each cycle for evaluation of potential TSU-68 biomarkers. Plasma levels of angiogenesis-related factors (vascular endothelial growth factor [VEGF], platelet-derived growth factor [PDGF]-AA, PDGF-AB, PDGF-BB, and fibroblast growth factor $[\mathrm{FGF}]$ ), apoptosis markers (caspasecleaved fragment of cytokeratin 18 [M30]), and inflammatory markers (C-reactive protein [CRP] and interleukin [IL]-6) were measured using an enzyme-linked immunosorbent assay according to the manufacturer's instructions (R\&D Systems, Minneapolis, MN).

The pharmacodynamics of TSU-68 was assessed by estimation of fold changes in plasma biomarker concentration from baseline to each cycle of the study treatment and their maximal changes during the entire study course. For correlative analysis of candidate plasma biomarkers, baseline plasma concentration and fold changes from baseline to the end of the first cycle of the study treatment were dichotomized according to the median values. In the patient subgroup categorized by baseline biomarker levels, PFSs were compared between the two arms to evaluate the impact of 
Table 1. Baseline characteristics

\begin{tabular}{|c|c|c|c|}
\hline Characteristic & TSU-68+docetaxel (n=38) & Docetaxel $(n=39)$ & p-value \\
\hline Age, median(range, yr) & $52(25-68)$ & $50(30-73)$ & 0.57 \\
\hline \multicolumn{4}{|l|}{ ECOG performance status } \\
\hline 0 & $21(55)$ & $19(49)$ & 0.65 \\
\hline $1-2$ & $17(45)$ & $20(51)$ & \\
\hline \multicolumn{4}{|l|}{ Menopausal status } \\
\hline Premenopausal & $11(29)$ & $16(41)$ & 0.34 \\
\hline Postmenopausal & $27(71)$ & $23(59)$ & \\
\hline \multicolumn{4}{|l|}{ Anthracycline sensitivity } \\
\hline Resistant & $13(34)$ & $12(31)$ & 0.81 \\
\hline Sensitive & $25(66)$ & $27(69)$ & \\
\hline HER2 status (IHC or FISH) & 33 & 39 & 0.74 \\
\hline Positive & $6(16)$ & $5(13)$ & \\
\hline Negative & $27(71)$ & $34(87)$ & \\
\hline Estrogen receptor status & 37 & 39 & 0.11 \\
\hline Positive & $26(70)$ & $20(51)$ & \\
\hline Negative & $11(30)$ & $19(49)$ & \\
\hline Progesterone receptor status & 37 & 39 & 0.49 \\
\hline Positive & $20(54)$ & $17(44)$ & \\
\hline Negative & $17(46)$ & $22(56)$ & \\
\hline \multicolumn{4}{|l|}{ Previous therapy } \\
\hline Surgery & $34(90)$ & $38(97)$ & 0.20 \\
\hline Hormonal therapy & $25(66)$ & $23(59)$ & 0.64 \\
\hline Radiotherapy & $21(55)$ & $26(67)$ & 0.35 \\
\hline \multicolumn{4}{|l|}{ Chemotherapy } \\
\hline Anthracycline & $38(100)$ & 39 (100) & $>0.99$ \\
\hline Taxanes & $8(21)$ & $8(21)$ & $>0.99$ \\
\hline
\end{tabular}

Values are presented as number (\%). ECOG, Eastern Cooperative Oncology Group; IHC, immunohistochemistry; FISH, fluorescence in situ hybridization.

baseline biomarker levels on the benefit of TSU-68. Analysis of fold changes in plasma biomarker concentrations from baseline across study treatment cycles was performed to determine the relationship with PFS in each treatment arm.

\section{Statistical analysis}

The chi-square test or Fisher exact test was used to determine the association between categorical variables, as appropriate. Log-transformation of the plasma biomarker concentration was performed for comparison. Comparisons of the means of biomarker-related variables between treatment groups and within groups were performed using the Student's $t$ test and Wilcoxon rank sum tests, respectively. The Kaplan-Meier method was used for estimation of the probability of survival, with comparison using a log-rank test. PFS was defined as the time between randomization and disease progression or death from any cause, whichever occurred first. Correlation analyses in terms of PFS were performed using baseline biomarker levels, and fold changes after the first cycle of study treatment. A p-value of $<0.05$ was considered statistically significant, and all analyses were performed using SPSS ver. 18.0 (SPSS Inc., Chicago, IL).

\section{Results}

\section{Baseline plasma biomarker levels}

Blood samples at baseline were available for all patients included in the phase II trial (38 for TSU-68 plus docetaxel and 39 for docetaxel alone). There were no significant differences in the baseline characteristics between the two groups (Table 1). The baseline levels of plasma biomarkers in the TSU-68 plus docetaxel and docetaxel-alone groups are summarized in Table 2 . There were no significant differences in 
the baseline plasma concentrations of biomarkers between the two treatment arms (Table 2, Fig. 1).

\section{Plasma biomarker dynamics with study treatment}

Maximal changes from baseline values were calculated for each biomarker using all evaluated values during serial measurements. Estimation and comparison of the ratios of the geometric mean of maximal biomarker changes in the TSU-68 plus docetaxel group to those in the docetaxel-alone group was performed to assess the magnitude of maximal changes in biomarkers according to the treatment arm (Fig. 2). However, no significant differences in the maximal changes in plasma levels of all evaluated biomarkers were observed between the two arms: CRP (ratio, 1,45; $\mathrm{p}=0.52$ ), FGF (0.97; $\mathrm{p}=0.89)$, IL-6 (0.88; $\mathrm{p}=0.80), \mathrm{M} 30(0.80 ; \mathrm{p}=0.34)$, PDGF-AA (1.18; $\mathrm{p}=0.08)$, PDGF-AB (1.06; $\mathrm{p}=0.68)$, PDGF-BB (0.73; $\mathrm{p}=0.11)$, and VEGF $(0.87 ; \mathrm{p}=0.34)$.

The fold changes after the first cycle of study treatment for each biomarker are summarized in Table 2. In the TSU-68 plus docetaxel group, the levels of CRP (median fold change, 1.5; $\mathrm{p}=0.002)$, PDGF-AA (1.3; $\mathrm{p}<0.001)$, PDGF-AB (1.1; $\mathrm{p}=0.003)$, and VEGF (1.1; $\mathrm{p}=0.001)$ were significantly increased at the end of the first cycle compared to those at baseline. In the docetaxel-alone group, there was a significant increase only in VEGF level (1.2; $\mathrm{p}=0.002)$.

\section{Baseline plasma biomarkers as a predictive factor}

In each subgroup categorized by the baseline biomarker levels ( $<$ median vs. $\geq$ median), PFS was compared between the TSU-68 plus docetaxel group and the docetaxel-alone group. In patients with low baseline PDGF-AA levels, patients treated with TSU-68 plus docetaxel had significantly worse median PFS than those treated with docetaxel alone (5.4 months [ $95 \%$ CI, 2.1 to 8.6 months] vs. 13.7 months [95\% CI, 5.5 to 21.9 months]; $\mathrm{p}=0.049$ ), whereas a trend toward a benefit on PFS was observed in the TSU-68 plus docetaxel group compared with the docetaxel-alone group in patients with high baseline PDGF-AA levels (9.7 months [95\% CI, 4.2 to 15.2 months] vs. 4.0 months [ $95 \% \mathrm{CI}, 0.0$ to 8.3 months]; $\mathrm{p}=0.18$ ) (Fig. 3). There was a significant interaction between the PFS benefit of TSU-68 and baseline PDGF-AA levels $(\mathrm{p}=0.03)$. Otherwise, no difference in PFS was observed between the two arms for all other comparisons in subgroups categorized by the baseline levels of all other candidate biomarkers $(p>0.1)$.

\section{Correlation between biomarker dynamics and PFS}

The association between the dynamics of plasma biomarkers and PFS was assessed in each treatment arm. For each biomarker, patients were grouped based on the median fold change from baseline to the end of the first cycle of the study treatment ( $<$ median vs. $\geq$ median). In patients treated with the combination of TSU-68 and docetaxel, PFS showed significant association with fold changes in CRP ( $p=0.001)$, IL-6 $(\mathrm{p}<0.001)$, PDGF-BB $(\mathrm{p}=0.02)$, and VEGF $(\mathrm{p}=0.047)$ following the first treatment cycle (Fig. 4). For all of these four biomarkers, patients with high fold changes had a better median PFS than those with low fold changes: CRP (15.8 months [95\% CI, 8.4 to 23.2 months] vs. 5.4 months [ $95 \%$ CI, 1.1 to 9.6 months]), IL-6 (18.9 months [95\% CI, 2.3 to 35.6 months] vs. 4.8 months [95\% CI, 1.5 to 8.1 months]), PDGF-BB (12.5 months [95\% CI, 6.0 to 19.0 months] vs. 5.4 months [95\% CI, 3.7 to 7.1 months]), and VEGF (15.8 months [95\% CI, 3.2 to 28.4 months] vs. 5.4 months [ $95 \%$ CI, 3.8 to 6.9 months]). In patients included in the docetaxel-alone arm, however, PFS was not related to the dynamics of all tested biomarkers after the first cycle: $C R P(p=0.41), F G F(p=0.99), I L-6(p=0.16)$, M30 $(\mathrm{p}=0.30)$, PDGF-AA ( $\mathrm{p}=0.73)$, PDGF-AB ( $\mathrm{p}=0.95)$, PDGF-BB $(\mathrm{p}=0.36)$, and VEGF $(\mathrm{p}=0.80)$.

\section{Discussion}

In our current study, we found that the activity of TSU-68, which inhibits VEGFR-2, PDGFR, and FGFR, might be affected by baseline plasma PDGF-AA levels. The dynamics of circulating soluble proteins that reflect angiogenesis and tumor microenvironmental status showed correlation with PFS in patients treated with TSU-68, but not in those who received cytotoxic chemotherapy alone. Our findings have implications for the clinical development of TSU-68 and the identification of potential biomarkers for TSU-68.

The combination of TSU-68 and docetaxel induced significant increases in the levels of CRP, PDGF-AA, PDGF-AB, and VEGF at the end of the first cycle compared to those at baseline, while VEGF was the only marker showing a significant increase with docetaxel. However, in comparison of the maximal magnitude of changes in biomarkers during the serial measurements, no significant difference in any biomarker was observed between the two groups. Previous studies have reported conflicting results regarding the pharmacodynamic effects of TSU-68 [4,5,8]. In several studies, no significant changes in angiogenesis-related plasma proteins were observed after treatment with TSU-68 [4,5]. Although a previous pharmacodynamic analysis of colorectal cancer patients treated with TSU-68 in combination with fluoropyrimidine and oxaliplatin reported a significant decrease in the PDGF series (PDGF-AA, AB, and BB) [8], it is unlikely that this result reflects the pharmacodynamic features of 
Table 2. Levels of biomarkers at baseline and their fold changes after cycle 1 of study treatment

\begin{tabular}{|c|c|c|c|c|c|c|c|}
\hline \multirow[b]{2}{*}{ Characteristic } & \multicolumn{3}{|c|}{ Baseline level } & \multicolumn{4}{|c|}{ Fold change after cycle 1} \\
\hline & $\begin{array}{l}\text { Docetaxel, } \\
\text { mean } \pm S D\end{array}$ & $\begin{array}{c}\text { TSU-68+docetaxel, } \\
\text { mean } \pm \text { SD }\end{array}$ & p-value & $\begin{array}{c}\text { Docetaxel, } \\
\text { median (IQR) }\end{array}$ & p-value $e^{a}$ & $\begin{array}{c}\text { TSU-68+docetaxel, } \\
\text { median (IQR) }\end{array}$ & p-value ${ }^{a)}$ \\
\hline $\mathrm{CRP}$ (mg/dL) & $1.36 \pm 2.59$ & $1.09 \pm 2.32$ & 0.29 & $1.2(0.3-4.9)$ & 0.1 & $1.5(0.7-6.8)$ & 0.002 \\
\hline FGF (pg/mL) & $6.53 \pm 3.97$ & $6.53 \pm 3.99$ & 0.95 & $1.0(0.9-1.3)$ & 0.33 & $1.0(0.9-1.3)$ & 0.52 \\
\hline IL-6 (pg/mL) & $7.82 \pm 13.72$ & $3.63 \pm 4.29$ & 0.30 & $0.6(0.3-1.2)$ & 0.23 & $0.7(0.3-1.5)$ & 0.52 \\
\hline M30 (U/L) & $366.89 \pm 614.59$ & $486.49 \pm 1,366.17$ & 0.88 & $0.9(0.7-1.2)$ & 0.22 & $0.9(0.5-1.1)$ & 0.09 \\
\hline PDGF-AA (pg/mL) & $3,287.00 \pm 1,327.31$ & $3,577.89 \pm 1,574.69$ & 0.43 & $1.1(0.9-1.3)$ & 0.09 & $1.3(1.0-1.4)$ & $<0.001$ \\
\hline PDGF-AB (pg/mL) & $24,534.03 \pm 10,548.00$ & $25,764.36 \pm 9,447.64$ & 0.49 & $1.0(0.9-1.2)$ & 0.99 & $1.1(0.9-1.4)$ & 0.003 \\
\hline PDGF-BB (pg/mL) & $2,799.00 \pm 1,463.33$ & $2,567.10 \pm 1,640.16$ & 0.31 & $1.0(0.8-1.3)$ & 0.84 & $1.1(0.8-1.3)$ & 0.28 \\
\hline VEGF (pg/mL) & $435.86 \pm 361.51$ & $388.27 \pm 238.25$ & 0.81 & $1.2(0.9-1.4)$ & 0.002 & $1.1(1.0-1.5)$ & 0.001 \\
\hline
\end{tabular}

$\mathrm{SD}$, standard deviation; IQR, interquartile range; CRP, C-reactive protein; FGF, fibroblast growth factor; IL, interleukin;

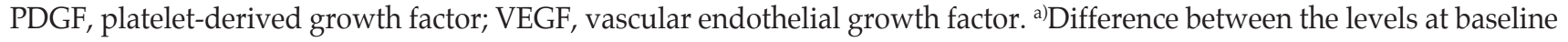
and after cycle 1 within the study group.

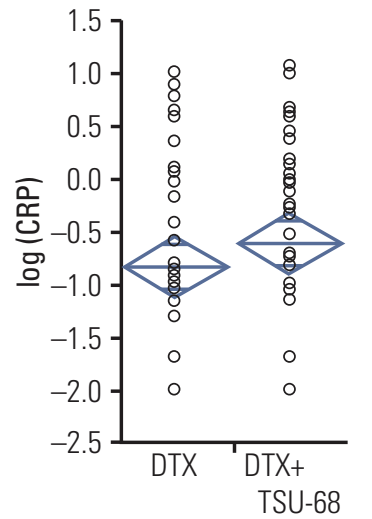

Treatment t test: $p=0.285$

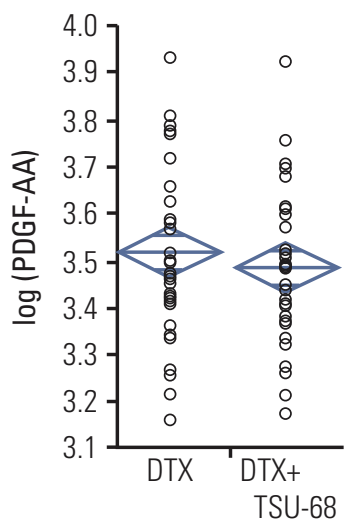

Treatment $t$ test: $p=0.433$
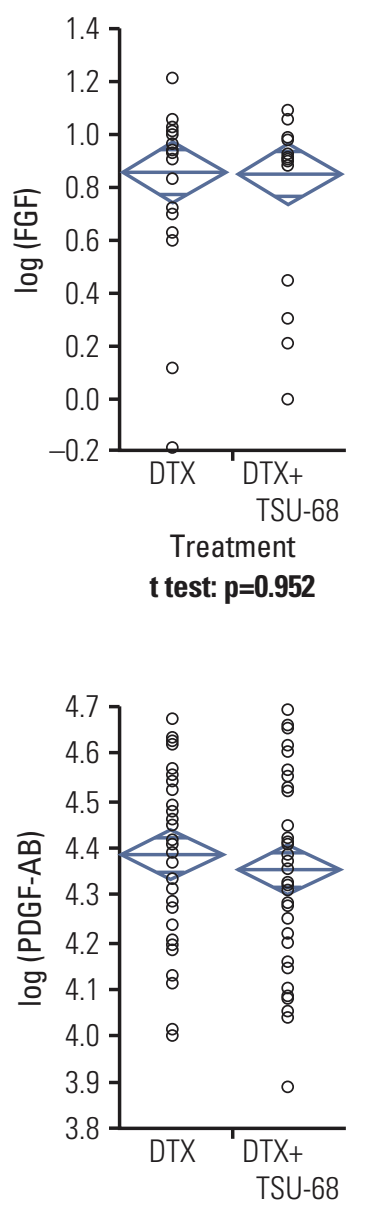

Treatment

t test: $p=0.485$

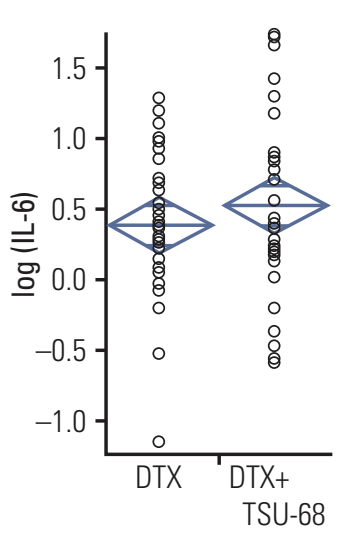

Treatment

t test: $p=0.302$

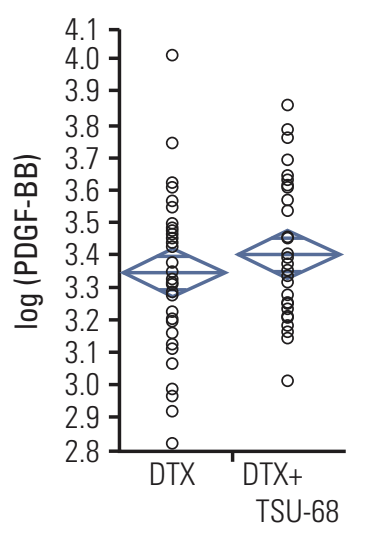

Treatment

t test: $p=0.311$

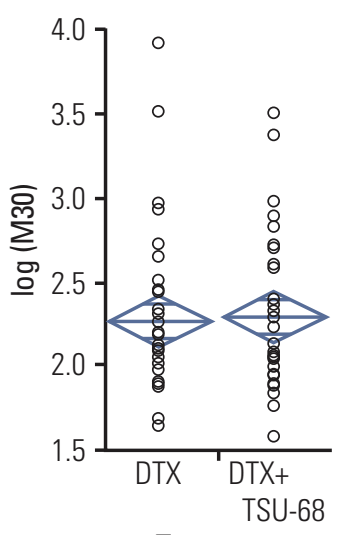

Treatment

t test: $p=0.875$

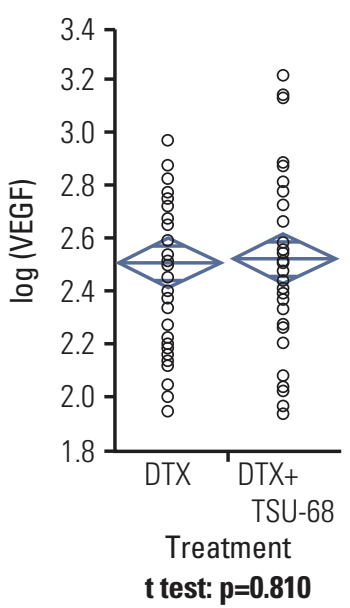

Fig. 1. Baseline levels of candidate plasma biomarkers between treatment arms. CRP, C-reactive protein; FGF, fibroblast growth factor; IL, interleukin; PDGF, platelet-derived growth factor; VEGF, vascular endothelial growth factor; DTX, docetaxel. 

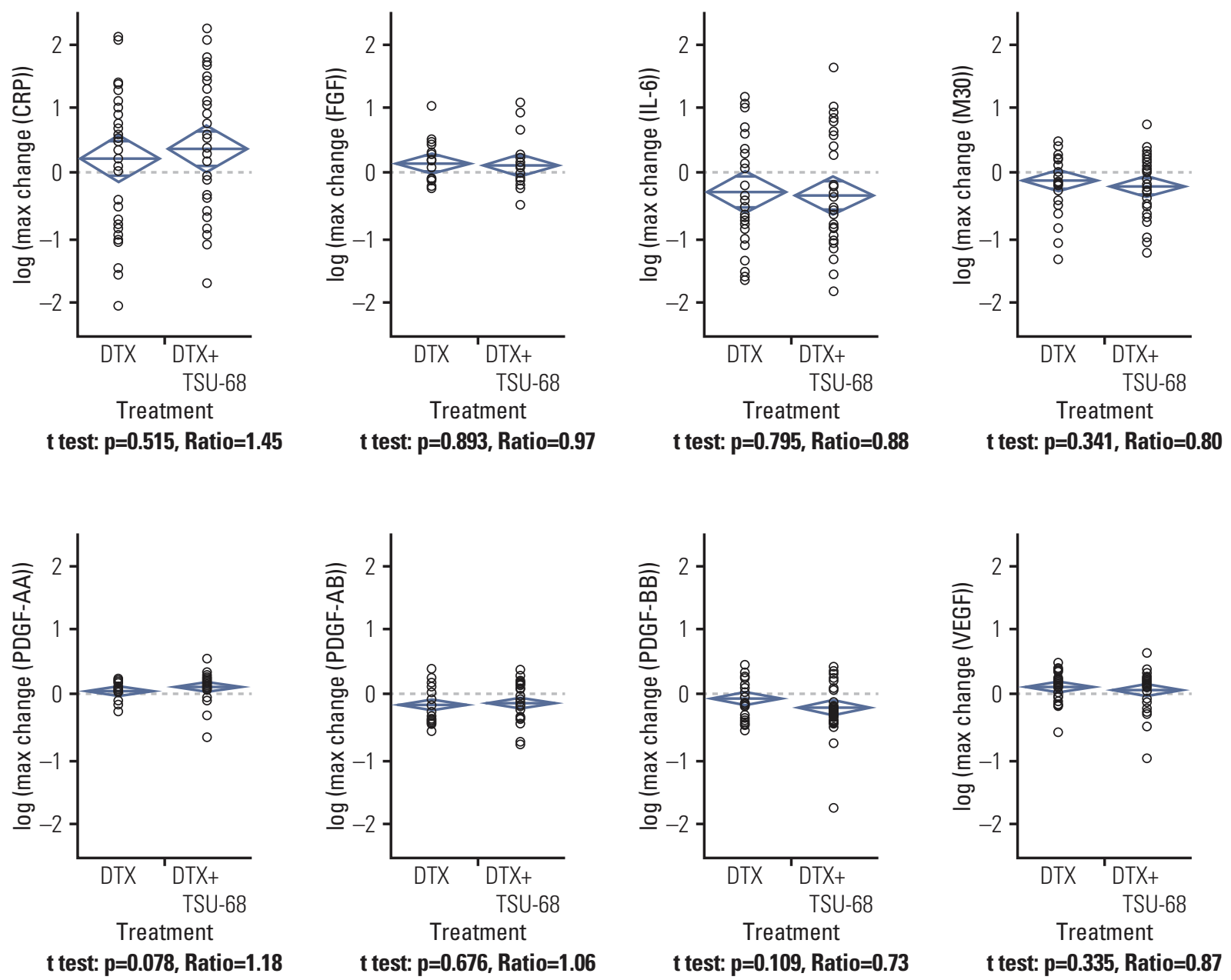

Fig. 2. Maximal fold changes in candidate plasma biomarkers during the entire study course between treatment arms. Ratio $=($ Geometric mean of maximal fold changes in the TSU-68 plus docetaxel group)/(Geometric mean of maximal fold changes in the docetaxel-alone group). CRP, C-reactive protein; FGF, fibroblast growth factor; IL, interleukin; PDGF, plateletderived growth factor; VEGF, vascular endothelial growth factor; DTX, docetaxel.

TSU-68 itself. Although it is possible that docetaxel confounded the effect of TSU-68 in our study, due to the randomized design of this trial, we were able to evaluate the pharmacodynamic impact of the addition of TSU-68 to docetaxel monotherapy. The limited impact of TSU-68 on pharmacodynamic markers in many trials, including ours, suggests that the current dose and schedule for TSU-68 may not be optimal to induce significant target inhibition and clinical efficacy.

In our primary analysis of this randomized phase II trial, TSU-68 plus docetaxel did not demonstrate superiority to docetaxel alone in terms of PFS [6]. In addition, when patients were categorized by clinical factors, including hormone receptors, HER-2 overexpression, and resistance to previous therapy, there was no subgroup benefit from combination therapy.

In our subanalysis of biomarker for TSU-68, we found that the efficacy of TSU-68 may depend on the pretreatment plasma concentration of PDGF-AA. The addition of TSU-68 appears to be associated with improved PFS in patients with low baseline PDGF-AA levels. However, a detrimental effect of TSU-68 was observed in patients with high baseline PDGF-AA levels, which contrasts with the expectation that PDGFR inhibition by TSU-68 may be more effective in patients with PDGF overexpression. This finding seems to be in line with the results of a previous randomized phase II study that compared TSU-68 with observation in patients with hepatocellular carcinoma [9]. Although the result was 


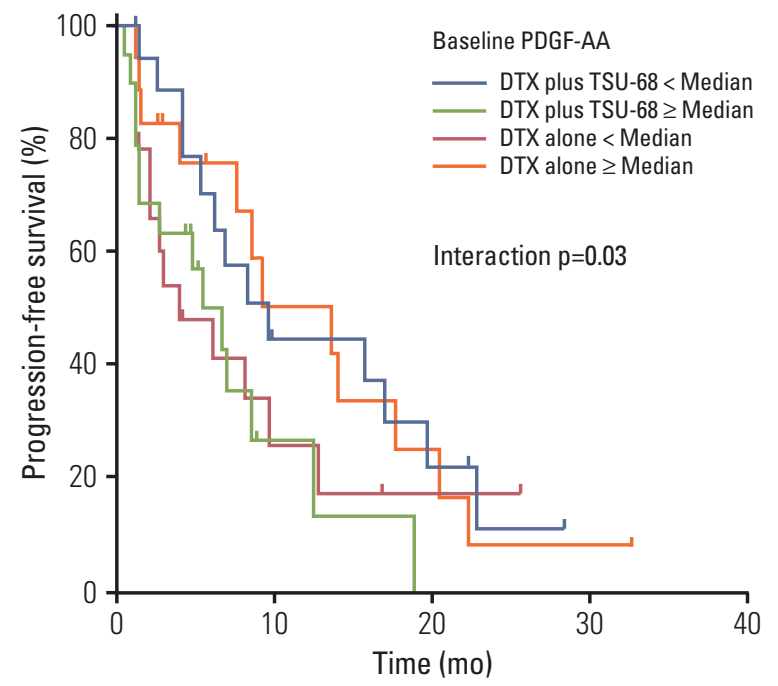

Fig. 3. Progression-free survival according to the baseline plasma platelet-derived growth factor-AA (PDGF-AA) levels. DTX, docetaxel.

not statistically significant, patients who received TSU-68 had a numerically longer PFS than those included in the observation arm in the subgroup with low baseline PDGFAA levels, whereas this trend was reversed in patients with high baseline PDGF-AA levels.

Until recently, investigation of the predictive role of circulating dimeric PDGF isoforms for antiangiogenic agents has been limited. Dimeric PDGF isoforms primarily induce paracrine stimulation of stromal fibroblasts and perivascular cells and also activate autocrine signaling for the proliferation of tumors [10]. However, the mechanism of the interaction between PDGF-AA and TSU-68 efficacy is unclear and will have to be defined in future studies. In many clinical trials for unselected patients with various types of cancer, TSU68 has not demonstrated a significant improvement in clinical outcomes $[6,9,11]$. Although the implication of PDGFAA as a predictive factor for TSU-68 should be validated in a large patient population, our results indicate that further investigations of TSU-68 for patients with relatively low expression of PDGF-AA will be necessary.

Monitoring of the dynamics of circulating protein biomarkers may provide the opportunity to predict clinical outcomes as well as achieve a better understanding of the mechanism of action of investigational drugs. In our current study, the dynamics of CRP, IL-6, PDGF-BB, and VEGF levels showed significant association with PFS in patients treated with TSU-68 plus docetaxel. This result confirms that the activity of TSU-68 is driven by the inhibition of angiogenesis and by its impact on the tumor microenvironment.
The relationship between the dynamics of plasma biomarkers and PFS was not seen in patients who received docetaxel monotherapy, thus the significance of our finding appears quite robust. A greater increase above the median in plasma VEGF (a median fold increase of 1.1) and PDGF-BB (1.1) following TSU-68 plus docetaxel was associated with better PFS. Given that inhibition of VEGFR and PDGFR results in a reactive increase in plasma VEGF and PDGF-BB $[4,12]$, this result may indicate that stronger VEGFR and PDGFR inhibition leads to a better PFS with TSU-68 treatment.

IL-6 is a proinflammatory marker and CRP is a wellknown indicator of inflammatory status $[13,14]$. Patients with fold changes above the median in CRP (the median of 1.5) and IL-6 (0.7) after treatment with TSU-68 had improved clinical outcomes in terms of PFS compared with those who showed less increased or decreased CRP and greater decrease of IL-6, respectively. Increases in these inflammatory markers can be regarded as a consequence of therapyinduced inflammation [13]. Death of cancer cells from anticancer treatment induces the release of necrotic products, leading to stimulation of cytokine-producing inflammatory cells. Currently, it is unclear whether therapy-induced inflammation is beneficial or harmful in cancer treatment [13]. The inflammation may stimulate residual cancer cells and help in regrowth via the activation of prosurvival genes; however, inflammation may also lead to activation of adaptive anti-tumor immune responses due to the increased presentation of tumor antigens. Further investigations will be needed in order to elucidate the effects of the dynamics of these biomarkers, which reflect the status of the tumor microenvironment, on the efficacy of antiangiogenic agents.

There are several limitations to our current study. Although the randomized design of this trial enabled comparisons of the impact of candidate biomarkers for TSU-68, our analysis was based on a small number of patients, limiting the ability of the multivariate analysis to adjust for potential confounding effects.

\section{Conclusion}

Results of our current exploratory analysis suggested that the baseline plasma concentration of PDGF-AA might be predictive of the efficacy of antiangiogenic agents, including TSU-68. As the addition of TSU-68 was detrimental in patients with high baseline PDGF-AA levels, this association should be validated in future studies and considered in the design of trials for the clinical development of TSU-68. A significant relationship between PFS with TSU-68 treatment and the dynamics of VEGF, PDGF-BB, CRP, and IL-6 confirmed 
A

B
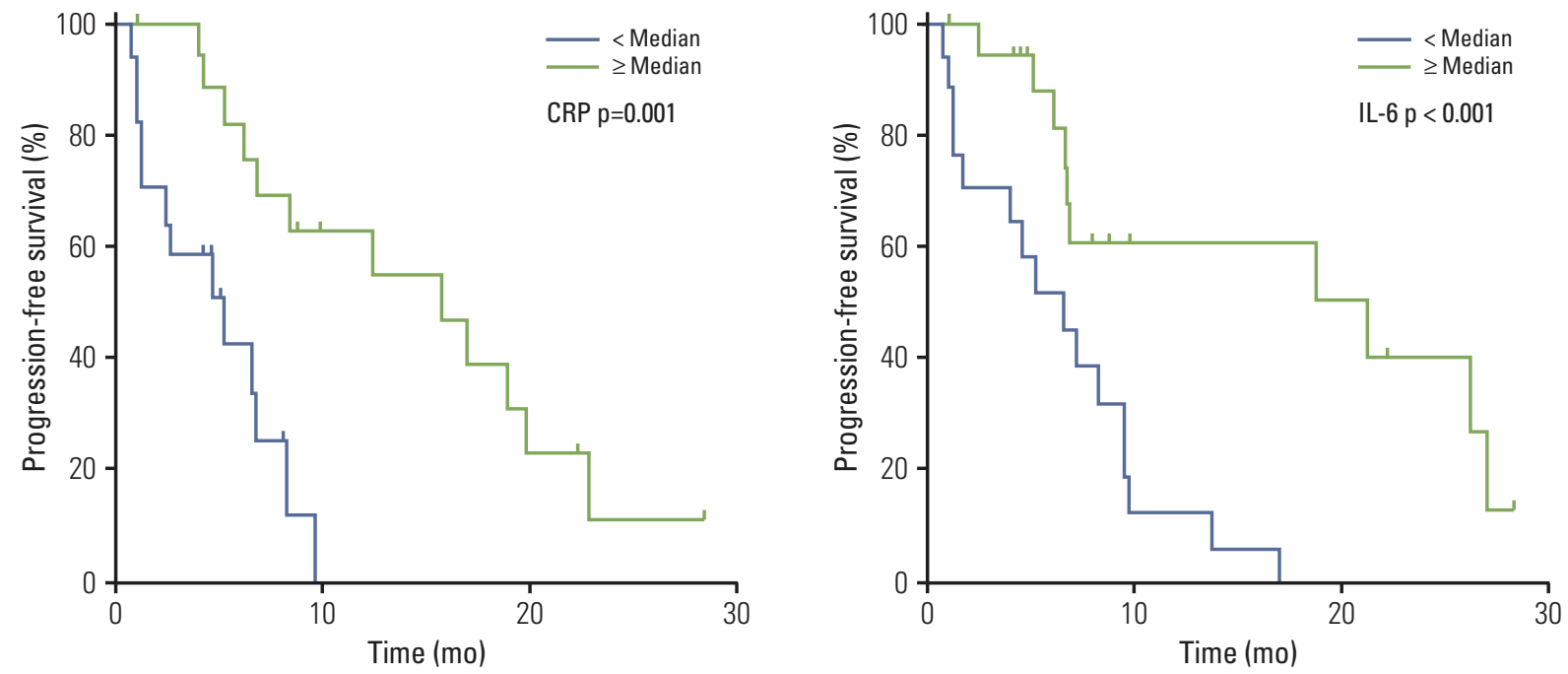

C

D
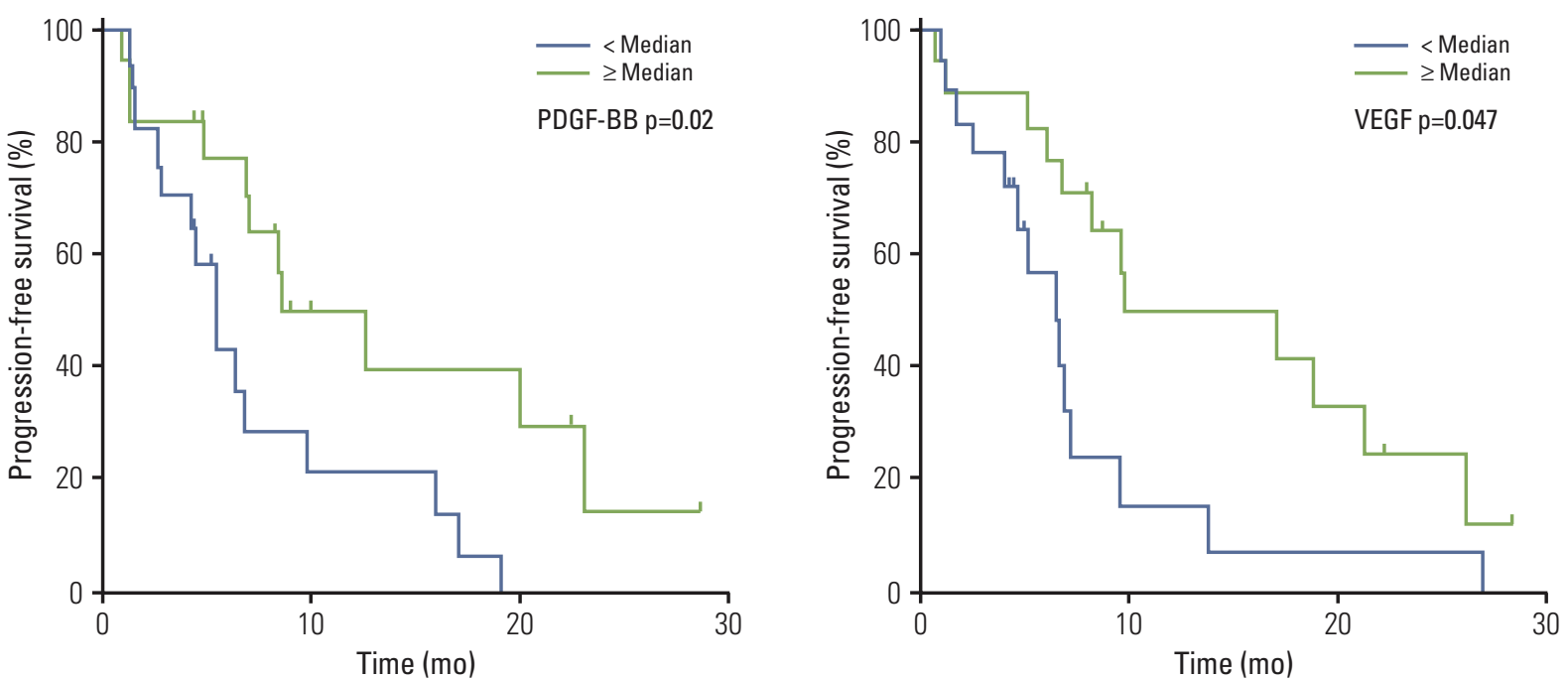

Fig. 4. (A-D) Association between progression-free survival and changes in plasma biomarkers from baseline to the end of the first cycle of the study treatment in the TSU-68 plus docetaxel group. CRP, C-reactive protein; IL, interleukin; PDGF, platelet-derived growth factor; VEGF, vascular endothelial growth factor.

that the efficacy of TSU-68 is due in part to the inhibition of angiogenesis/lymphangiogenesis as well as the inflammatory reaction, and also suggested that interim monitoring of these markers may be useful for prediction of outcomes.

\section{Conflicts of Interest}

This study was supported by Taiho Pharmaceutical Co., Ltd. 


\section{References}

1. Hicklin DJ, Ellis LM. Role of the vascular endothelial growth factor pathway in tumor growth and angiogenesis. J Clin Oncol. 2005;23:1011-27.

2. Reddy S, Raffin M, Kaklamani V. Targeting angiogenesis in metastatic breast cancer. Oncologist. 2012;17:1014-26.

3. Laird AD, Vajkoczy P, Shawver LK, Thurnher A, Liang C, Mohammadi M, et al. SU6668 is a potent antiangiogenic and antitumor agent that induces regression of established tumors. Cancer Res. 2000;60:4152-60.

4. Suzuki Y, Saeki T, Aogi K, Toi M, Fujii H, Inoue K, et al. A multicenter phase II study of TSU-68, a novel oral multiple tyrosine kinase inhibitor, in patients with metastatic breast cancer progressing despite prior treatment with an anthracycline-containing regimen and taxane. Int J Clin Oncol. 2013; 18:590-7.

5. Toi M, Saeki T, Iwata H, Inoue K, Tokuda Y, Sato Y, et al. A multicenter phase II study of TSU-68, an oral multiple tyrosine kinase inhibitor, in combination with docetaxel in metastatic breast cancer patients with anthracycline resistance. Breast Cancer. 2014;21:20-7.

6. Kim SB, Yoo C, Ro J, Im SA, Im YH, Kim JH, et al. Combination of docetaxel and TSU-68, an oral antiangiogenic agent, in patients with metastatic breast cancer previously treated with anthracycline: randomized phase II multicenter trial. Invest New Drugs. 2014;32:753-61.

7. Murukesh N, Dive C, Jayson GC. Biomarkers of angiogenesis and their role in the development of VEGF inhibitors. Br J Can- cer. 2010;102:8-18.

8. Shin SJ, Jung M, Jeung HC, Kim HR, Rha SY, Roh JK, et al. A phase I pharmacokinetic study of TSU-68 (a multiple tyrosine kinase inhibitor of VEGFR-2, FGF and PDFG) in combination with S-1 and oxaliplatin in metastatic colorectal cancer patients previously treated with chemotherapy. Invest New Drugs. 2012;30:1501-10.

9. Inaba $Y$, Kanai F, Aramaki T, Yamamoto T, Tanaka T, Yamakado K, et al. A randomised phase II study of TSU-68 in patients with hepatocellular carcinoma treated by transarterial chemoembolisation. Eur J Cancer. 2013;49:2832-40.

10. Pietras K, Sjoblom T, Rubin K, Heldin CH, Ostman A. PDGF receptors as cancer drug targets. Cancer Cell. 2003;3:439-43.

11. Koizumi W, Yamaguchi K, Hosaka H, Takinishi Y, Nakayama N, Hara T, et al. Randomised phase II study of S-1/ cisplatin plus TSU-68 vs S-1/ cisplatin in patients with advanced gastric cancer. Br J Cancer. 2013;109:2079-86.

12. Ebos JM, Lee CR, Christensen JG, Mutsaers AJ, Kerbel RS. Multiple circulating proangiogenic factors induced by sunitinib malate are tumor-independent and correlate with antitumor efficacy. Proc Natl Acad Sci U S A. 2007;104:17069-74.

13. Grivennikov SI, Greten FR, Karin M. Immunity, inflammation, and cancer. Cell. 2010;140:883-99.

14. Pierce BL, Ballard-Barbash R, Bernstein L, Baumgartner RN, Neuhouser ML, Wener MH, et al. Elevated biomarkers of inflammation are associated with reduced survival among breast cancer patients. J Clin Oncol. 2009;27:3437-44. 